\title{
La distribution spatiale des précipitations et son rôle dans la transformation pluie-débit
}

\author{
Ch. Obled Y. Rodriguez \\ Institut de mécanique, ENSHMG-INPG, Grenoble
}

\section{Introduction}

On se propose d'analyser ici le rôle de la distribution spatiale de la pluie dans le bassin versant sur la réponse de ce dernier. La précipitation, cumulée sur un pas de temps adéquat, est un processus continu et la surface associée présente une morphologie variée plus ou moins lisses.

Notre objectif final serait de trouver des variables, ou index, caractéristiques de cette morphologie, et susceptibles d'influer sur la relation pluie-débit. La première variable, largement étudiée dans le passé, est la pluie moyenne sur le bassin. Mais nous souhaitons y ajouter d'autres indices traduisant sa plus ou moins grande uniformité, comme nous le définirons plus loin. Nous gardons à l'esprit que nous souhaitons des variables globales, caractéristiques de ce qui se passe sur tout le bassin, et susceptibles d'être introduites dans des modèles du type hydrogramme unitaire, DPFT, ARMAX, etc.

Dans une première partie, nous reviendrons sur les moyens de définir de telles variables et sur les problèmes que cela peut soulever. Dans la seconde partie, nous mettrons en regard ces informations avec des hydrogrammes de débits. Nos exemples seront pris sur le bassin pilote du Gardon d'Anduze $\left(550 \mathrm{~km}^{2}\right)$, objet de nombreuses études antérieures et pour lequel un pas de temps de 1 heure semble convenable. Il est l'objet de modélisations de type hydrogramme unitaire (B. VERSIANI, 1983) dans lesquelles on voudrait tenir compte de cette dynamique des pluies pour améliorer la prévision. Cette dynamique pourrait ultérieurement être apprécier par radar, et une expérience est en cours dans ce sens (J.D. Creutin et al., dans cette même réunion). On rappelle sur la figure 1, la localisation du bassin et des stations disponibles dans et au voisinage du bassin.

\section{Caractérisation de la répartition spatiale des pluies et sensibilité aux erreurs}

\subsection{Méthodologie utilisée}

On ne reviendra pas sur les techniques d'ajustement de surface, largement étudiées par Creutin (1979), Tourasse (1981) et LEBEL (1984), et résumées récemment par OBLED (1986). Dans le cas d'un réseau suffisamment dense, les résultats de ces techniques diffèrent peu. Nous nous limiterons à l'une des plus simples à mettre en œuvre, cele des fonctions splines "plaque mince".

\section{Additional results concerning the spatial distribution of rainfall and its role in the transformation of rain-flowrate}

In this preliminary study, the calculation of time pitch isohyet surfaces are firstly recalled and then its sensitivity as regards the uncertainty of localization of stations, which is finally not critical in normal conditions, is analysed. Then the emphasis is placed on the importance of being fully familiar with rainfall spatial distribution so as identify the individual responses of sub-basins by selecting the irregular intervals when they have been excited in various ways by the input of rain. To conclude, the emphasis is placed on the size, even with average rainfall, between highly concentrated isohyets, or rather distributed, on the discharge response. These somewhat original observations, obtained in the Gardon d'Anduze $\left(500 \mathrm{~km}^{2}\right)$, are currently being modelized. 

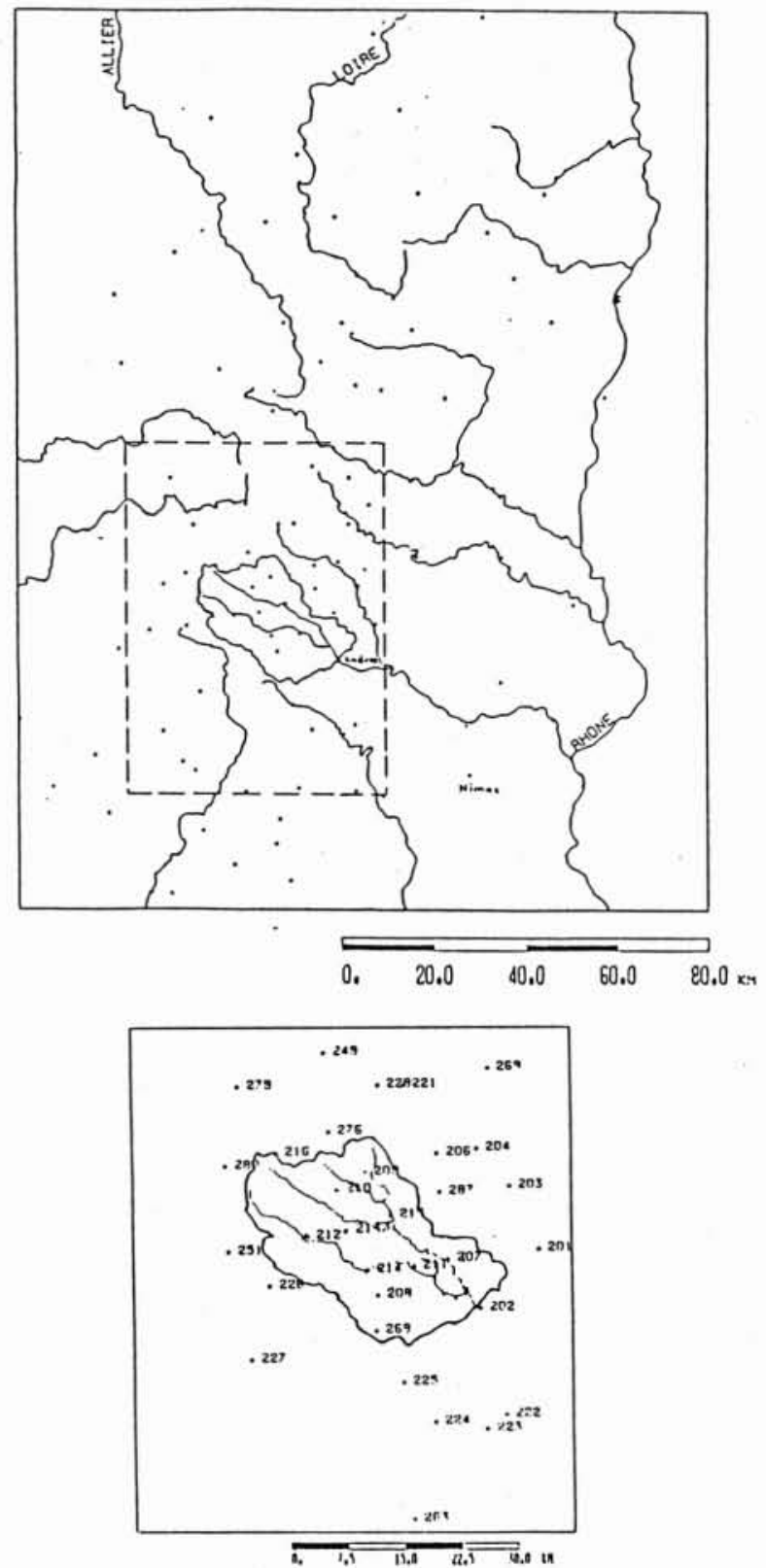

1. Situation du gardon d'Anduze et localisation des stations pluviométriques.

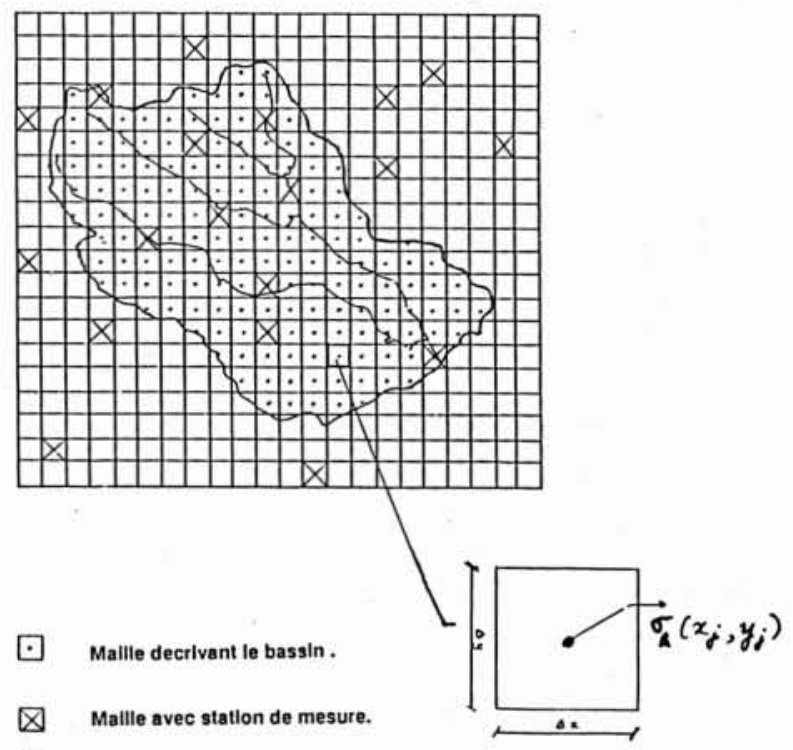

2 . Exemple de maillage dans un bassin.

On la trouvera détaillée dans Le Hucher (1986). Elle fournit, à partir d'un ensemble de stations $S_{i}$ de coordonnées $x_{i}, y_{i}$, où la pluie observée est notée $Z_{i}(k)$ pour l'événement $k$, une surface analytique:

$$
\begin{aligned}
\sigma_{k}(x, y)= & \alpha_{k} x+\beta_{k} y+\gamma_{k}+\Sigma \lambda_{i}(k)\left\{\left(x-x_{i}\right)^{2}\right. \\
& \left.\left.+\left(y-y_{i}\right)^{2}\right\} \log \sqrt{(x}-x_{i}\right)^{2}+\left(y-y_{i}\right)^{2}
\end{aligned}
$$

qui interpole les pluies observées: $\sigma_{k}\left(x_{i}, y_{i}\right)=z_{i}(k)$.

La lame d'eau moyenne sur le bassin s'exprime théoriquement :

$$
Z M_{k}=1 / B \int_{B} Z_{k}(x, y) d x d y
$$

Elle sera estimée sur la surface ajustée par:

$$
\mathrm{ZM}_{\mathrm{k}}=1 / B \int_{B} \sigma_{k}(x, y) d x d y
$$

et cette intégrale est calculée numériquement par :

$$
Z M_{k}=1 / M \sum_{j=1}^{M} \sigma_{k}\left(x_{j}, y_{j}\right)
$$

où $M$ est le nombre de mailles carrées discrétisant la surface $B$ (fig. 2).

On trouvera chez T. LEBEL (1984) des développements sur les incertitudes liées à cette estimation. En particulier, on a beaucoup travaillé sur le rôle des erreurs de mesure de $Z_{i}(k)$ sur l'incertitude de $Z M_{k}$.

Préoccupé par les valeurs moyennes, on a moins travaillé sur les réseaux d'isohyètes, car :

- pour les réseaux denses, ils diffèrent très peu selon la technique employée (cf. LEBEL, 1984);

- pour les réseaux lâches, ils dépendent de la méthode (et du réseau), mais l'estimation de $Z M$ restait néanmoins assez stable.

Par contre, pour caractériser la morphologie du champ pluvieux, le réseau d'isothyètes estimé sera l'ingrédient essentiel. Si le réseau est dense, il sera peu sensible à la méthode. Si les erreurs de mesure de $Z_{i}(k)$ sont raisonnables, les isohyètes $\mathrm{y}$ sont peu sensibles aussi. Mais une autre source d'erreur est apparue.

\subsection{Incertitude de positionnement des stations}

Curieusement, il est difficile d'obtenir de certains services la position exacte de leur station, ce pour diverses raisons pratiques. Il est bien sûr possible de refaire un levé exhaustif sur le terrain, mais auparavant, on a voulu en évaluer l'intérêt. Pour cela, on a considéré que les erreurs de localisations étaient de la forme :

$$
x_{i}=x_{i}+\delta x_{i} \quad y_{i}=y_{i}+\delta y_{i}
$$

les $x_{i}$ et $y_{i}$ étant les vraies coordonnées, et $\delta x_{i}$ et $\delta y_{i}$ les erreurs que l'on suppose issues d'une loi normale $N\left(0, \sigma_{\delta}\right)$ avec $\sigma_{\delta}=1$ ou $2 \mathrm{~km}$. Ces valeurs sont raisonnables, surtout pour notre réseau où les distances entre stations sont de 5 à $10 \mathrm{~km}$, et où l'incertitude concerne la localisation dans un village ou entre le bourg et un hameau.

Pour tester cette influence, nous avons recalculé, sur un certain nombre d'événements : 
a) les valeurs moyennes sur des sous-bassins

On en donne ici un exemple pour l'épisode du 11-09-76, où l'on a considéré deux sous-bassins et les pluies moyennes supérieures à $0,5 \mathrm{~mm}$. On s'aperçoit (tableau 1) que les fluctuations dues aux variations de localisation du réseau (avec $\sigma_{6}=1$ et $2 \mathrm{~km}$ ) n'induisent que des fluctuations de 5 à 10 , et 10 à $15 \%$ respectivement, largement incluses dans l'incertitude d'échantillonnage (due à la densité du réseau) sur les valeurs moyennes.

Tableau 1

Effet de l'incertitude de localisation sur le calcul des pluies moyennes sur des bassins

\begin{tabular}{|c|c|c|c|c|}
\hline \multirow[b]{2}{*}{$\begin{array}{c}\text { Bassin des } \\
\text { Gardons }\end{array}$} & \multirow[b]{2}{*}{$\begin{array}{c}\text { Superficie } \\
\mathrm{km}^{2}\end{array}$} & \multicolumn{3}{|c|}{ Erreurs de localisations $x, y$} \\
\hline & & $\sigma_{b}=1$ & $\sigma_{b}=2$ & $\begin{array}{c}\text { Fluctuations } \\
\text { en } \%\end{array}$ \\
\hline Anduze & 550 & $\begin{array}{l}6,2 \\
5,5\end{array}$ & $\begin{array}{l}10,1 \\
10,4\end{array}$ & \\
\hline Mialet & 237 & $\begin{array}{l}6,9 \\
5,7\end{array}$ & $\begin{array}{l}12,9 \\
10,2\end{array}$ & \\
\hline Saint Jean & 265 & $\begin{array}{l}9,3 \\
7,0\end{array}$ & $\begin{array}{l}16,9 \\
13,1\end{array}$ & \\
\hline
\end{tabular}

Les fluctuations calculées heure par heure et par bassin fournissent une moyenne et un écart-type calculés sur les heures de l'épisode oủ $Z M>0,5 \mathrm{~mm}$.

\section{b) les réseaux d'isohyètes}

En effet, on pourrait craindre que les valeurs moyennes restent stables, mais que les réseaux d'isohyètes, qui nous servirons à calculer noyaux pluvieux, épicentres, etc., ne soient profondément modifiés.

On donne, pour un épisode contenant beaucoup de variabilité spatiale (fig. 3 , p. suivante), les réseaux d'isohyètes obtenus avec la localisation des stations telle qu'elle nous a été fournie, puis avec des perturbations $\sigma_{\delta}$ $=1$ et $2 \mathrm{~km}$.

On constatera que qualitativement, les déformations des isohyètes sont mineures et que leur allure reste très reconnaissable.

Et cela pour des incertitudes assez considérables, affectant toutes les stations, alors que dans la réalité, seules quelques stations sont incertaines à moins de $1 \mathrm{~km}$.

En conclusion, sur le réseau de mesures considéré, les incertitudes liées au caractère aléatoire du champ pluvieux, étudiées antérieurement, ou les incertitudes de localisations des points de mesure, n'empêchent pas d'avoir une perception satisfaisante de la distribution des pluies. Nous pouvons donc envisager de la caractériser.

\subsection{Caractérisation de la distribution spatiale de la pluie}

Pour cela, nous suivons deux approches selon que l'on privilégie la topographie du champ pluvieux ou celle du bassin.

\subsubsection{Découpage en sous-bassins}

Dans ce dernier cas, nous découpons le bassin $B$ en sous-bassins plus petits $B_{j}$ (en nombre limité, $j=2$ à 5 ) sur lesquels nous calculons des pluies moyennes pour chaque pas $k$ :

$$
Z M_{k}\left(B_{j}\right) \text { exprimées en } \mathrm{mm} \text {. }
$$

La comparaison entre les valeurs de ces sous-bassins et la valeur moyenne d'ensemble montre implicitement si la pluie s'est répartie de façon très disparate, et localise éventuellement le sous-bassin concerné. Malheureusement, les sous-bassins ne sont pas toujours de taille comparable, et la moyenne n'a alors plus le même sens. D'autre part, le système pluvieux peut présenter un épicentre marqué mais être à cheval sur deux sousbassins, auquel cas les moyennes ne reflètent pas l'existence de l'épicentre.

\subsubsection{Caractérisation du réseau d'isohyètes}

D'où un autre approche, peut-être plus globale, qui considère la forme du système pluvieux dans le bassin global à travers des indices d'uniformité, ou de concentration, de la pluie. Pour cela, nous avons défini deux indices :

\section{a) la précipitation "Supseuil "}

Pour différents seuils préfixés $(5,10,15 \mathrm{~mm}$ en 1 heure), on calcule le volume de pluie tombée $P S$ sur le bassin en excès du seuil choisi $S P$, par:

$$
P S(S P)=1 / B \int_{B} \operatorname{Sup}[0,((x, y)-S P)] \cdot d x d y
$$

ou en pratique : $=1 / M \sum_{i=1}^{L}\left(\left(x_{i}, y_{i}\right)-S P\right)$

sur les $L$ points où $(x, y)>S P, M$ points définissant le bassin total.

\section{b) la surface "Supseuil "}

Il s'agit alors seulement de déterminer la superficie du bassin ayant reçu plus de $S P \mathrm{~mm}$, d'où la variable : $S \%(S P)$ exprimée en $\%$ de la surface du bassin.

On envisage aussi, ultérieurement, de déterminer la position de cette zone par rapport à l'exutoire, ou au réseau de drainage.

Dans nos exemples, nous avons travaillé avec des mailles de $2 \times 2 \mathrm{~km}$. Pour chaque seuil $S P$, on constitue évidemment la série chronologique $S \%(S P)$ des pourcentages de surface atteinte heure par heure lors des différents épisodes. 
$\sigma_{\delta}=1 \mathrm{~km}$.
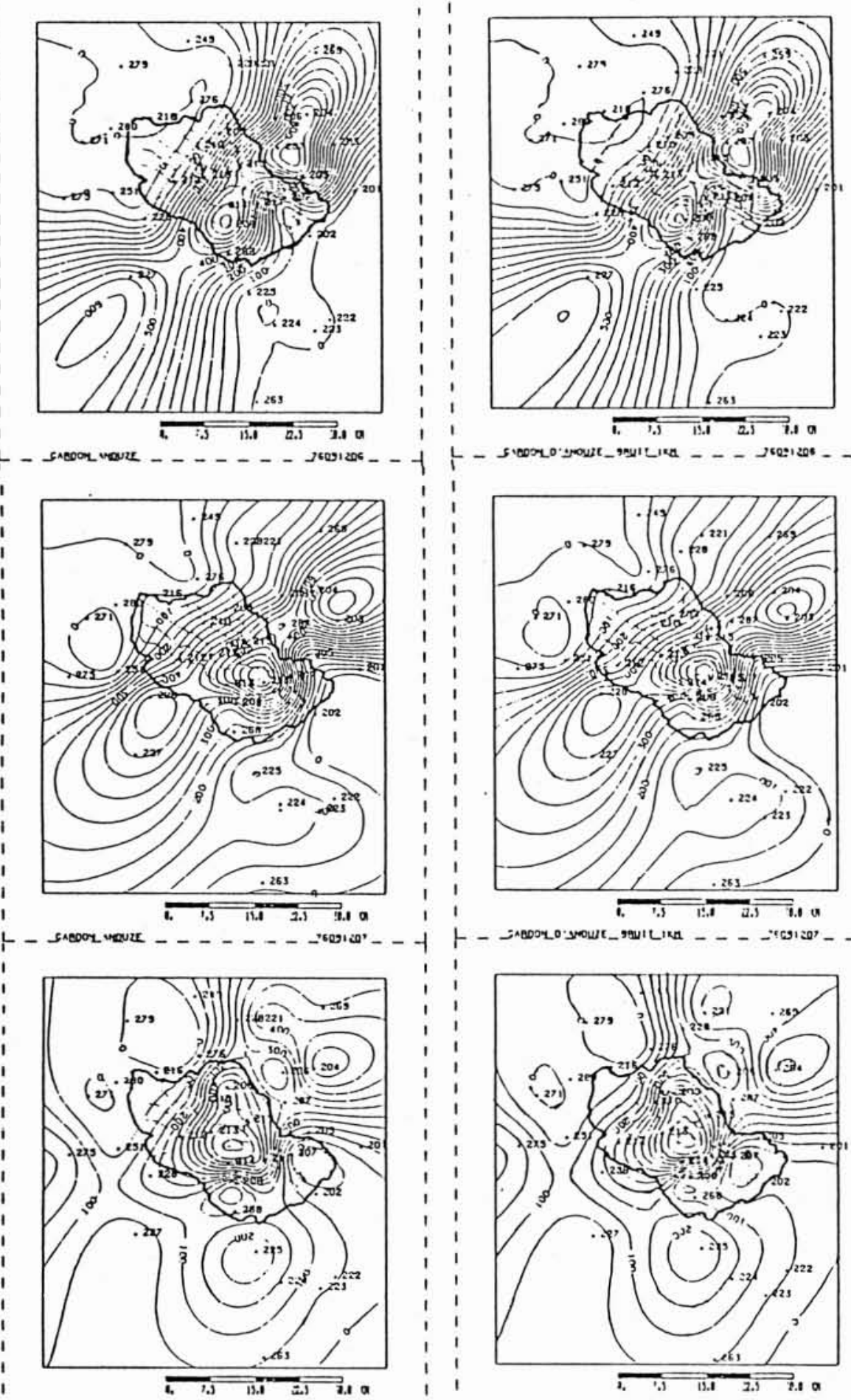

sesoon vele -

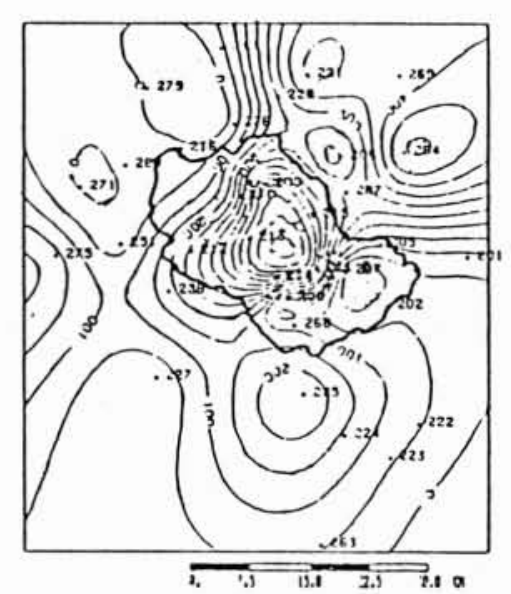

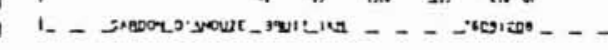

$\sigma_{\delta}=2 \mathrm{~km}$.

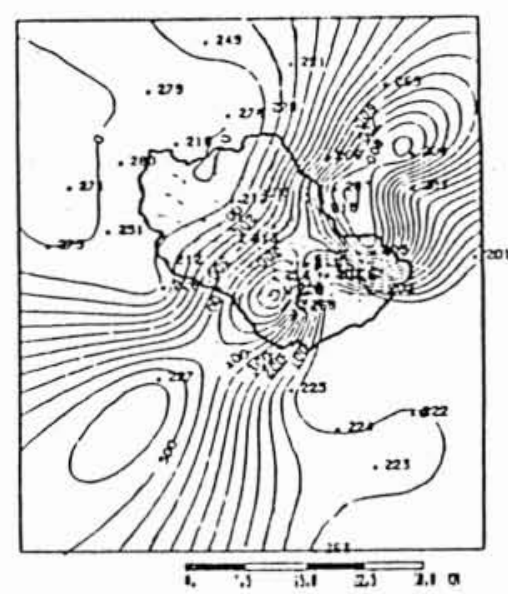

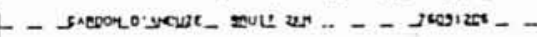

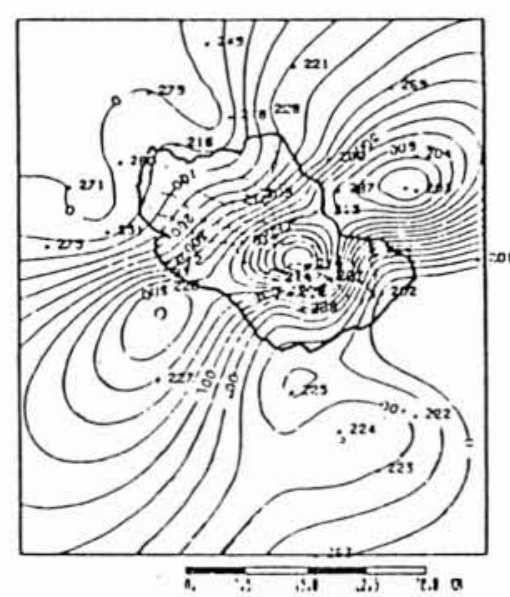

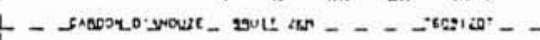

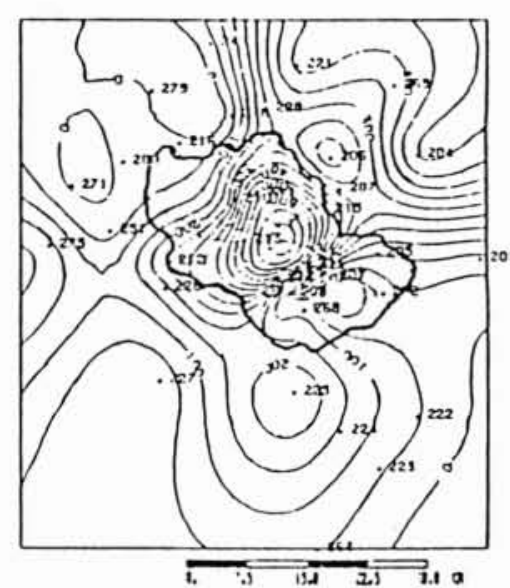

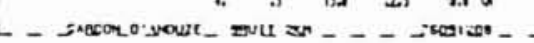

3. Réseau d'isohyètes avec et sans perturbations. 


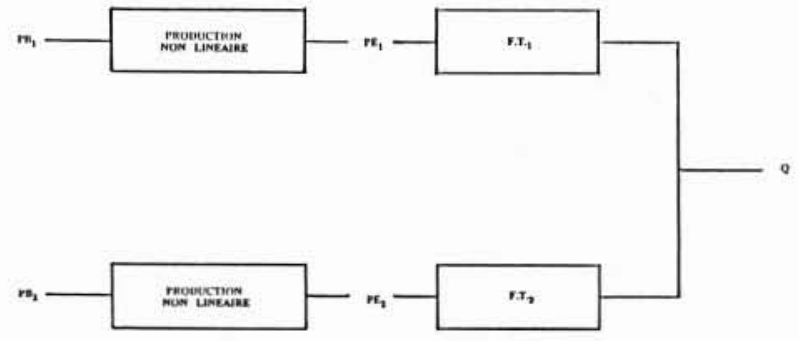

4. Schéma de modélisation multi-entrée.
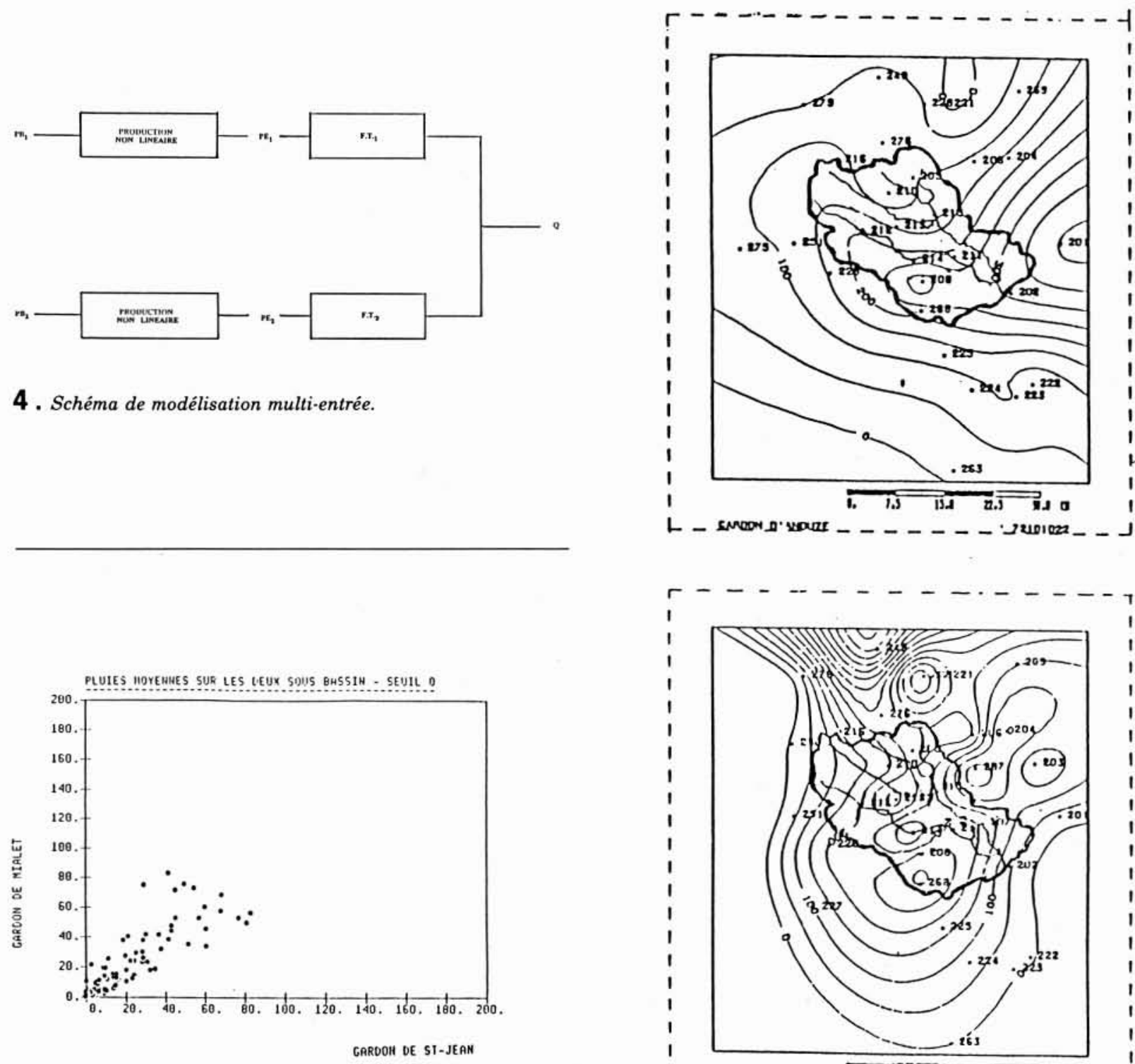

5. Nuages de corrélation entre les sous-bassins pour l'épisode du 13.10.86.

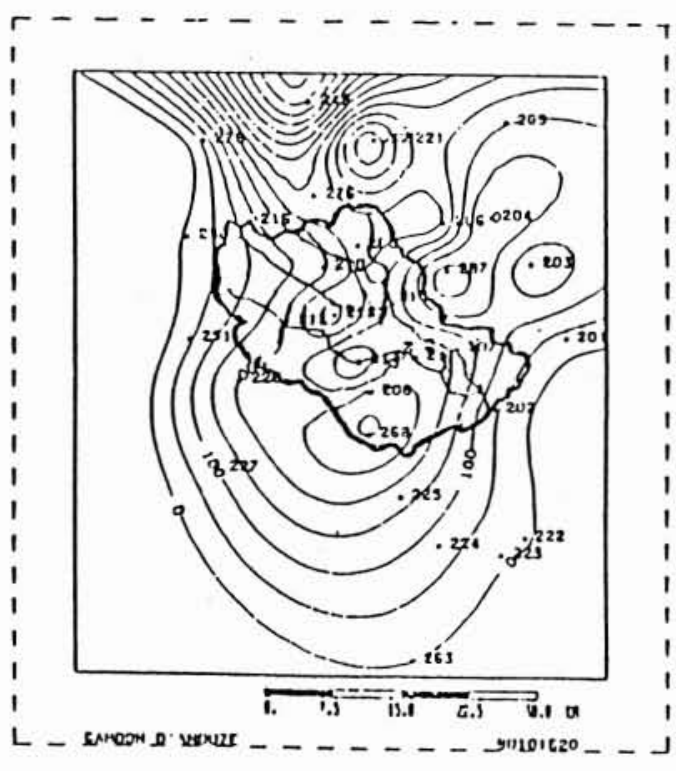

2. Tentatives de prise en compte de la répartition spatiale dans des modèles globaux pluies-débits

Ici encore, nous aurons deux approches qui privilégieront l'une le bassin, l'autre le système pluvieux.

\subsection{Identification des réponses individuelles des sous-bassins}

Nous restons dans l'optique de l'hydrogramme unitaire en proposant, pour deux sous-bassins ou plus, d'identifier leur réponse unitaire propre, et les pluies efficaces correspondantes, par les techniques de la DPFT. Le modèle répond donc au schéma de la figure 4 .

Pour avoir quelques espoirs de différencier ces réponses, il faut proposer des épisodes dont la distribution spatiale de la pluie a été la plus hétérogène possible, et donc où les sous-bassins ont été excités aussi différemment que possible. On a alors des chances d'identifier les réponses distinctes des sous-bassins.

La sélection des épisodes s'est faite en corrélant les séries $Z M_{1}(k)$ et $Z M_{2}(k)$ par épisode, et en ne retenant que les épisodes mal corrélés entre les deux sous-bassins.

On trouve aussi une douzaine d'épisodes, dont un exemple est donné figure 5 :

Sur ces données, la modélisation DPFT avec 2 entrées pluies est en cours.

On notera cependant que deux moyennes spatiales $Z M$ quasiment identiques sur le bassin global peuvent provenir de systèmes pluvieux très différents (cf. fig. 6). 

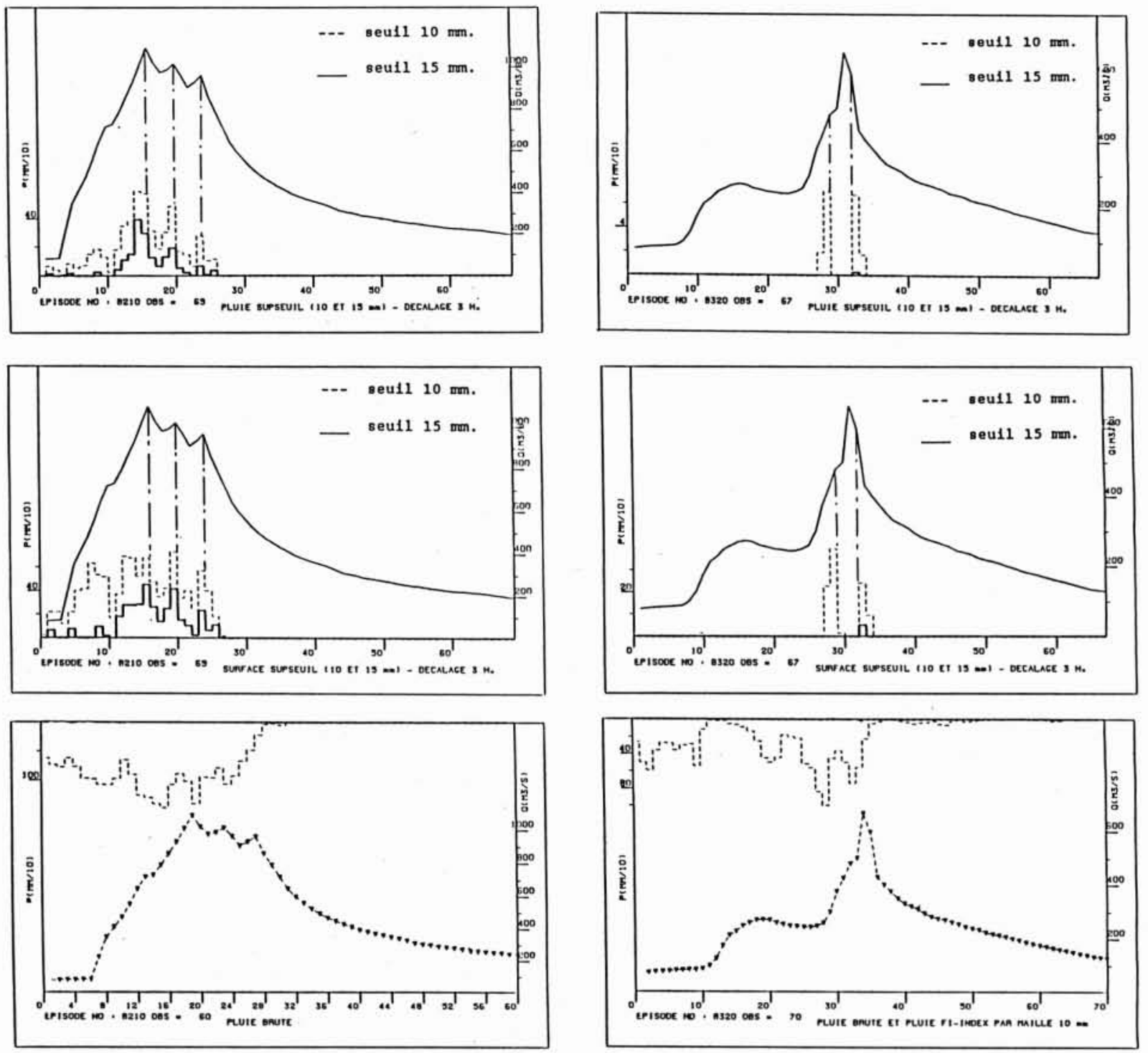

7. Episode du 6.11.82.

8. Episode du 14.12.83.

\subsection{Morphologie de l'épisode pluvieux et écou- lement : analyse préliminaires.}

Il s'agit donc ici d'une approche qui privilégie plutôt la topographie du champ pluvieux. Elle consiste à mettre en regard, de manière qualitative, les différentes chroniques de pluie Supseuil $P S_{k}(S P)$ et surface Supseuil $S \%_{k}$ $(S P)$ avec les débits $Q_{k}$ pour différents seuils $S P$ convenablement choisis, de 10 à $20 \mathrm{~mm} / \mathrm{h}$.

On note, à un décalage près de 3 à 5 heures, que pour $S P=10$ à $15 \mathrm{~mm} / \mathrm{h}$, les impulsions du signal $S \%$ se retrouvent bien dans les pics de l'hydrogramme. Cela est même frappant sur l'épisode du 06-11-82 comportant 3 pics successifs (figure 7). Par contre, l'analogie est un peu moins bonne avec les pluies Supseuil, mais surtout avec la pluie moyenne sur le bassin.

Il est difficile de conclure trop rapidement sur un petit nombre d'épisodes. Mais il semble bien que l'on puisse proposer une modélisation du bassin avec:

- une composante rapide, qui expliquerait les pics seuls et serait très sensible aux fortes intensités localisées;

- une composante plus lente, qui expliquerait la moyenne de l'onde de crue, et qui serait plus sensible aux volumes écoulés. Attention, il ne s'agit pas là du " débit de base ", car cette composante "lente" que nous évoquons apparait parfois seule (cf. épisode du 14-12-83 sur la figure 8 ) peut quand même faire passer les débits de 50 à $300 \mathrm{~m}^{3} / \mathrm{s}$.

\subsection{Aperçu sur une modélisation élémentaire de la relation pluie brute-pluie efficace}

Si l'on abandonne les aspects physiques suggérés par le paragraphe précédent, on peut chercher quand même à rendre plus objectif la transformation de la pluie brute (ici la pluie moyenne sur le bassin, ou plutôt, le champ de pluie) en pluie efficace.

En effet, nous disposons, pour l'ensemble des épisodes qui ont été traités en DPFT, d'une série présumée de pluies efficaces (cf. figure 9 ) obtenues par déconvolution.

Une idée consisterait à trouver, à chaque pas de temps $k$, le seuil de pluie $S P(k)$ tel que la pluie observée, sur les maille où elle est supérieure à ce seuil, fournisse la pluie efficace de ce pas de temps. Soit dans nos notations : 


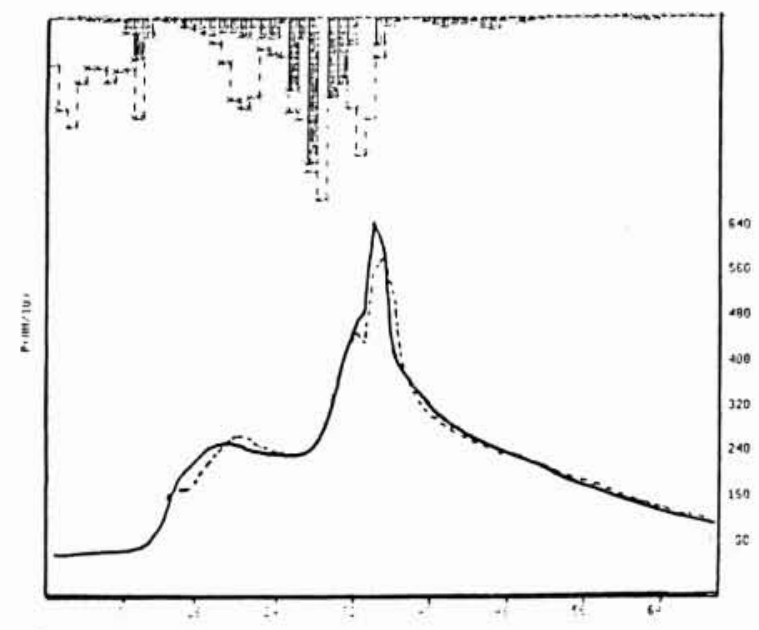

9.

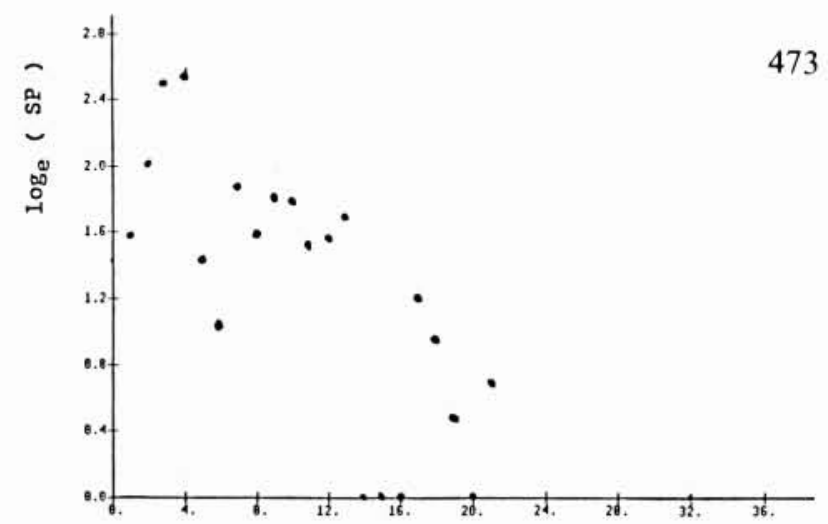

10. Evolution du seuil dans une crue monopic pour avoir $P S_{k}(S P(k))=P E_{k}$

\section{$S P(k) \quad$ tel que $P S_{k}(S P(k))=P E_{k}$}

C'est donc une forme d'estimation de la "capacité d'infiltration " moyenne du bassin au temps $k$. En fait, c'est plutôt cette capacité dans la zone de pluies intenses, en supposant le bassin relativement homogène du point de vue de l'infiltration.

Appliquée à des crues monopic, on observe sur la figure 10 cependant bien une décroissance exponentielle de ce seuil, comme le propose la plupart des modèles.

\section{Discussion et conclusion}

Sur le plan phénoménologique, ces résultats alimentent le débat actuel sur les mécanismes du ruissellement.

Si les modélisations actuelles tentent de prendre en compte la variabilité des paramètres d'infiltration, elles ignorent en général celle de la pluie. Certes les concepts Hortoniens ne sont plus pris au pied de la lettre, et le concept des zones sources semble désormais bien adapté aux régions présentant un relief marqué (cf. travaux de MÉrot et de AmBroise, 1986). Mais, dans les régions à influence méditerranéenne, les intensités de pluie sont telles qu'elles constituent une $3^{\mathrm{e}}$ source de génèse du ruissellement, comme l'ont montré des essais sous simulateur de pluie, et sans que les mécanismes en soient complètement élucidés (cf. par exemple Cosandey, 1986, et Muxart et al., 1987).

Sur le plan pratique, si l'on se contraint pour des raisons opérationnelles à une approche globale, il semble cependant possible de prendre en compte grossièrement, mais de manière plus satisfaisante que par une pluie moyenne, la répartition spatiale des pluies et les zones de fortes intensités.

Adresse des auteurs

Messieurs Ch. Obled et Y. Rodriguez

$I M G$

BP 68

Domaine universitaire

38402 St Martin d'Hères

Tél. : (16) 76.44.82.63

\section{Références}

AMBroise B., 1986. - Rôle hydrologique des surfaces saturées en eau dans le bassin du Ringelbach à Soultzeren (Hautes Vosges). Colloque sur l'environnement. Université du Rhin Supérieur. Strasbourg. Septembre 1986.

Andrieu H., Creutin J.D., Delrieu G., Fournay J. et Pointin Y., 1987. - Hydrologie radar : Bilan et expérimentations en cours ". SHF. Comité technique. Session $N^{0} 135$.

Cosandey C., 1986. - De l'origine de l'écoulement rapide de crue dans un petit bassin versant forestier breton. $Z$. Geomorph. N.F. Suppl.-Bd 60, Berlin-Stuttgart, pp. 177-186.

CREUTIN J.D., 1979. - Méthode d'interpolation optimale de champs hydrométéorologiques. Comparaison et application à une série d'épisodes pluvieux cévénosl. Thèse de DocteurIngénieur. USMG-INPG. Grenoble.

LeBel T., 1984. - Moyenne spatiale de la pluie sur un bassin versant: estimation optimale, génération stochastique et gradex des valeurs extrêmes. Thèse de Docteur-Ingénieur. USMG-INPG. Grenoble.

LEHUCher P.M., 1986. - Etudes des épisodes pluvieux intenses sur la région Provence-Côte d'Azur-Riviera Italienne (1974-1982). Cartographie et reconstitution des séries horaires sur le bassin du Réal-Colobrier et du Paillon. Thèse de Docteur de l'INPG. Grenoble. 199 p.

Muxart T., Cosandey C., Billard A. et Valadas B., 1987. Dynamique des versants et occupation humaine dans les Cévennes (Montagne de Bull. Assoc. Géogr. Franç. Paris. 1987-1.

OBLED C., 1986. - Introduction au Krigeage pour les Hydrologues. $2^{\mp}$ Journéees Hydrologiques ORSTOM-Montpellier. Septembre 1986. Colloques et Séminaires. ORSTOM Ed. pp. 174-222.

TOURAsse P., 1981. - Analyse spatiale et temporelle de précipita tions et utilisation dans un système de prévision de crues. Application à la région Cévenole. Thèse de Docteur-Ingénieur. USMG-INPG. Grenoble.

Versiani B., 1983. - Modélisation Pluie-Débit pour la prévision des crues. Thèse de Docteur-Ingénieur. USMG-INPG. Grenoble.

Remerciements: Cette étude a été menée avec la collaboration de $\mathrm{M}^{\text {lle }}$ Bosc $\mathrm{M}$. qui a travaillé dans notre équipe dans le cadre de son projet de fin d'études de l'E.N.T.P.E. 
M. OBLED: Un éclaircissement. Vous parlez de pluies « intenses ", or toutes les pluies considérées dans cette salle sont intenses. En fait, il faut considérer l'intensité moyenne sur le bassin et les intensités ponctuelles sur les différentes zones de bassin. Nous pouvons avoir des réponses différentes du bassin pour deux épisodes de même intensité moyenne. Mais il semble que lorsque la pluie est concentrée, le "dôme" d'intensité maximale répond de manière différente, ce qui mérite d'être pris en compte par rapport à une pluie de même volume, mais d'intensité uniforme.

$M^{m e} \operatorname{COSANDEY}:$ Là encore, tout dépend des processus qui génèrent le ruissellement, et pour lesquels l'état de recouvrement du sol par la végétation a une grande importance. Si le sol est totalement couvert, je pense que la répartition des intensités, dans le temps et dans l'espace, joue un rôle relativement secondaire. Il en est tout autrement si le sol est nu ou mal couvert des phénomènes de modifications de l'état de surface peuvent se produire sous l'impact des gouttes d'eau, et l'intensité de la pluie joue un rôle essentiel : dans ce cas, non seulement la valeur de l'intensité maximum en chaque site intervient, mais également la position de cette intensité dans la chronologie des événements pluvieux. Ainsi, pour deux précipitations identiques quant à l'état du bassin versant, la durée, la hauteur totale, l'intensité maximum, le coefficient de ruissellement pourra être très différent si les fortes intensités se produisent d'abord, elles peuven modifier l'état de surface de telle sorte que les précipitations suivantes, d'intensité moindre, ruissellent; si elles se produisent en fin d'épisode, les premières pluies de plus faible intensité peuvent s'infiltrer sur un sol dont la porosité de surface est conservée.

De même, l'intensité moyenne d'une précipitation à l'échelle du bassin versant n'aura pas les mêmes conséquences suivant que cette intensité est la résultante d'intensités localement très différentes.

M. Le Président : Il s'agit de tirer de l'observation des éléments à introduire par la suite dans le modèle applicable au bassin.

M. $M$. ROCHE : Des études analogues ont été faites il y a une trentaine d'années par Braquaval, d'E.D.F., sur le bassin du Mayoukouré (1957-58).

M. P.A. ROCHE : Ce qui est surprenant est que, ces phénomènes étant bien connus depuis longtemps, on ait toujours des difficultés à les prendre en compte à l'intérieur des modèles. Est-ce que grâce à l'accumulation de ces expériences, on ne pourrait pas essayer de travailler dans la prise en compte de ce facteur d'intensité ?
M. M. ROCHE : Je peux dire pourquoi ceci a été abandonné. Ce n'est pas une question de mesures. La méthode facilitait grandement l'analyse du ruissellement. Par contre, on ne l'a jamais vraiment utilisée au niveau de la synthèse, quand on voulait répartir des définitions statistiques ou des caractéristiques trouvées sur des observations de longue durée, pour reproduire les faits observés, en utilisant les moyens de l'époque. Actuellement, avec les progrès de la modélisation, on pourrait reconsidérer la question.

M. OBLED : Dernier complément, nous espérons que ces index - que nous essayons de reconstituer assez laborieusement à partir des mesures au sol - pourront, s'ils arrivent à prouver leur intérêt dans la modélisation des débits, être acquis à moindre coût à l'aide d'outils comme le radar, calibrés à partir des postes synchronisés. Nous pourrions alors effectuer très rapidement toute cette cartographie.

M. LEVIANDIER : L'intensité de la pluie peut être assez facilement prise en compte en diminuant le pas de temps du modèle, ce qui a pu être vérifié, quoique de façon imparfaite, sur l'Orgeval, en passant de 1 jour à $1 / 12$ de jour.

M. RodRIGUez: Dans notre cas, sur les Cévennes, le pas de temps est d'une heure. Réduire le pas de temps accroît l'incertitude sur la qualité de la mesure de la pluie. Je voudrais ajouter que nous cherchons à développer des modèles statistiques qui intègrent ces différents index, de manière à pouvoir prendre en compte l'intensité de la pluie lors de son transfert en débit. Dans des modèles simples de type "fonction de transfert", vous transférez une quantité d'eau plus ou moins grande de la même façon. Or, dans notre cas, plus l'intensité dans le bassin est forte, plus ce transfert se fait rapidement. D'un temps de montée moyen de 6 heures, nous passons, pour les fortes intensités, à 4 heures. De plus, il semble y avoir des régimes différents. Nous cherchons à discerner le moment où le régime prépondérant est dû à la réponse des nappes, et où il est dû à un ruissellement sur les surfaces recevant les fortes intensités.

M. DUBAND: Au sujet de la délimitation du pas de temps, il y a un problème pratique : c'est celui du synchronisme des enregistrements, car les postes sont relevés indépendamment les uns des autres. Sur les pluviographes et limnigraphes, l'incertitude sur les relevés peut atteindre une à deux heures. Seule l'acquisition en temps réel avec des horloges réglées permet la synchronisation des observations pluviographiques et des observations limnimétriques. Actuellement, il y a une limite sur le pas de temps en dessous de laquelle nous ne pouvons pas descendre. 